\title{
Interactive Effects of Dorsomedial Hypothalamic Nucleus and Time-Restricted Feeding on Fractal Motor Activity Regulation
}

\author{
Men-Tzung Lo ${ }^{1,2 *}$, Wei-Yin Chiang ${ }^{1}$, Wan-Hsin Hsieh ${ }^{1}$, Carolina Escobar ${ }^{3}$, Ruud M. Buijs ${ }^{4}$ \\ and $\mathrm{Kun} \mathrm{Hu}^{1 *}$ \\ ${ }^{1}$ Medical Biodynamics Program, Division of Sleep and Circadian Disorders, Brigham and Women's Hospital, Harvard \\ Medical School, Boston, MA, USA, ${ }^{2}$ Institute of Translational and Interdisciplinary Medicine and Department of Biomedical \\ Sciences and Engineering, National Central University, Taoyuan, Taiwan, ${ }^{3}$ Departamento de Anatomía, Facultad de Medicina, \\ Edificio "B" 4 ํ Piso, Universidad Nacional Autónoma de México, México, Mexico, ${ }^{4}$ Departamento de Biología Celular y \\ Fisiología, Instituto de Investigaciones Biomédicas, Universidad Nacional Autónoma de México, México, Mexico
}

One evolutionary adaptation in motor activity control of animals is the anticipation of food that drives foraging under natural conditions and is mimicked in laboratory with daily scheduled food availability. Food anticipation is characterized by increased activity a few hours before the feeding period. Here we report that $2-h$ food availability during the normal inactive phase of rats not only increases activity levels before the feeding period but also alters the temporal organization of motor activity fluctuations over a wide range of time scales from minutes up to $24 \mathrm{~h}$. We demonstrate this multiscale alteration by assessing fractal patterns in motor activity fluctuations - similar fluctuation structure at different time scales - that are robust in intact animals with ad libitum food access but are disrupted under food restriction. In addition, we show that fractal activity patterns in rats with ad libitum food access are also perturbed by lesion of the dorsomedial hypothalamic $(\mathrm{DMH})$ - a neural node that is involved in food anticipatory behavior. Instead of further disrupting fractal regulation, food restriction restores the disrupted fractal patterns in these animals after the DMH lesion despite the persistence of the 24-h rhythms. This compensatory effect of food restriction is more clearly pronounced in the same animals after the additional lesion of the suprachiasmatic nucleus (SCN) - the central master clock in the circadian system that generates and orchestrates circadian rhythms in behavior and physiological functions in synchrony with day-night cycles. Moreover, all observed influences of food restriction persist even when data during the food anticipatory and feeding period are excluded. These results indicate that food restriction impacts dynamics of motor activity at different time scales across the entire circadian/daily cycle, which is likely caused by the competition between the food-induced time cue and the light-entrained circadian rhythm of the SCN. The differential impacts of food restriction on fractal activity control in intact and $\mathrm{DMH}$-lesioned animals suggest that the $\mathrm{DMH}$ plays a crucial role in integrating these different time cues to the circadian network for multiscale regulation of motor activity.

Keywords: motor activity, food anticipation, fractal regulation, circadian rhythm, suprachiasmatic nucleus, dorsomedial hypothalamic nucleus 


\section{INTRODUCTION}

One of the most important external stimuli that influence locomotor activity control is food availability. When food is provided to animals only during a fixed and limited time period each day (i.e., time-restricted feeding), animals will show foodanticipatory activity (FAA) after 5-7 days, which is characterized by increased activity levels $(1-3 \mathrm{~h})$ just before the feeding period (Mistlberger, 1994; Stephan, 2002; Silver et al., 2011). Once FAA is established, it will take several days before FAA completely disappears when food restriction is omitted, and it will reappear at the same time of the day when animals are food deprived again. FAA is believed to be controlled by an intrinsic food entrained oscillator or oscillatory network with a circadian period close to $24 \mathrm{~h}$ (Escobar et al., 2009). This is evident from the fact that FAA occurs only when the cycle of restricted food availability is close to $24 \mathrm{~h}$ or harmonics of $24 \mathrm{~h}$ such as $12 \mathrm{~h}$ (Mistlberger et al., 2012; Patton et al., 2014), e.g., FAA is absent when the cycle period is $<20 \mathrm{~h}$ (Boulos et al., 1980; Mistlberger and Marchant, 1995; Takasu et al., 2012). However, the evaluation of FAA has been exclusively focused on mean activity levels a few hours before and/or throughout the feeding period and on the circadian/daily rhythmicity of FAA. How time-restricted feeding affects dynamics of motor activity at different times of the daily cycle and at different time scales is largely unknown.

A wide range of biological functions from cellular processes to overt system behavior display endogenous circadian rhythms of $\sim 24 \mathrm{~h}$ that are regulated by the circadian timing system, a network consisting of a huge number of cellular autonomous circadian clocks in the brain and peripheral organs (Weaver, 1998; Reppert and Weaver, 2002). In mammals, the master clock of the circadian system is located in the hypothalamic suprachiasmatic nucleus (SCN), which orchestrates circadian rhythms in behavior and physiology through numerous neural and humoral feedback loops (Sakamoto et al., 1998; Weaver, 1998; Yamazaki et al., 2000). In addition to circadian/daily rhythms in physiological functions, the SCN also impacts motor activity at different time scales from minutes to hours (PittmanPolletta et al., 2013). Lesioning the SCN in animals not only abolishes circadian rhythm of motor activity but also disrupts the temporal organization of motor activity fluctuations that normally display fractal patterns-similar temporal structure and statistical properties at different time scales ( $\mathrm{Hu}$ et al., 2007). Moreover, we found recently that interrupting the normal synchronization between endogenous circadian rhythms and behavioral cycles can also disrupt fractal activity patterns in animals with intact SCN, resembling the effect of the SCN lesion (Hsieh et al., 2014). Together these results provide strong evidence that the circadian control network plays an essential role in activity regulation at multiple time scales rather than only at a single time scale of $\sim 24 \mathrm{~h}$.

There is evidence for the mechanistic interactions between FAA and SCN-enforced circadian oscillations. For instance, circadian clock genes, such as Cry1, Cry2, and Bmal1, that are critical for the SCN function at neural level also play essential roles in regulating FAA rhythms (Takasu et al., 2012); and the SCN and its interaction with the dorsomedial hypothalamic (DMH) nucleus, another important neural node that influences motor activity though not generating circadian rhythms, contribute to the intensity of FAA (Gooley et al., 2006; Mieda et al., 2006; Angeles-Castellanos et al., 2010; AcostaGalvan et al., 2011; Merkestein et al., 2014) though neither the SCN nor the DMH is required for the existence of FAA (Landry et al., 2006; Mistlberger et al., 2009; Moriya et al., 2009). In addition, we have shown that the SCN activity, as measured by vasopressin release or with c-Fos, is inhibited when animals exhibit FAA (Kalsbeek et al., 1998; Acosta-Galvan et al., 2011). Thus, it is possible that time-restricted feeding can affect the temporal structure of motor activity via its influences on SCN activity and the circadian system. Here we hypothesize that time-restricted feeding not only induces FAA but also affects motor activity control at multiple time scales, altering fractal activity patterns. We further hypothesize that the multiscale effect of time-restricted feeding on motor activity control is via its influence on the component in the circadian network underlying food entrainment. Thus, we test whether the effect of time-restricted feeding on fractal activity patterns is different in animals with the sequential lesions of the DMH and the SCN. Note that time-restricted feeding during the normal inactive phase induces a time cue "conflicting" to the endogenous circadian time of the SCN. For animals without the SCN, it is possible that time-restricted feeding can have different influences on multiscale activity regulation as compared to those in animals with the intact SCN. Specifically, we expect that time-restricted feeding in animals after the SCN lesion enhances daily activity rhythm and, thus, helps to restore disrupted fractal activity patterns in these animals.

\section{MATERIALS AND METHODS}

\section{Ethics Statement}

All animal experiments were approved by the ethical committee at the Instituto de Investigaciones Biomédicas performed according to the guide for care and use of animal experimentation in Universidad Nacional Autónoma de México, which conforms to international guidelines for animal handling.

\section{Animals and Protocols}

To test our hypothesis, we studied locomotor activity of 18 Wistar rats that were placed in individual cages under 12:12 LD cycles. Animals were divided into three groups. (Group 1) Six rats (controls) were not disturbed with ad libitum food access for 2-4 weeks. (Group 2) Six rats underwent a 16-day scheduled food restriction protocol in which food was only available at Zeitgeber time (ZT) 6-8 h during the light phase. The experimental procedures were highly controlled to ensure that only food availability was the only different factor between the two experimental protocols for Group 1 (i.e., with ad libitum food access) and Group 2 (i.e., with food only available in a restricted period every day), respectively. (Group 3) The other six animals underwent two sequential surgeries for the lesions of $\mathrm{DMH}$ and SCN, respectively (see details below). After each surgery, animals went through the same protocol with ad libitum food access as 
the animals in Group 1 for at least 12 days and then the same 16-day food restriction protocol as the animals in Group 2.

\section{Lesions}

For the DMH or SCN lesion, animals were anesthetized with 200 $\mu \mathrm{L}$ Ketamine $(10 \mathrm{mg} / 100 \mathrm{~g})$ and $165 \mu \mathrm{L}$ Xilazine $(1 \mathrm{mg} / 100 \mathrm{~g})$ and mounted in a stereotaxic frame. Lesions were aimed to the DMH or the SCN with either kainic acid (a bilateral injection of $1 \%$ kainic acid, $100 \mathrm{~nL}$ Sigma) or with electrolytic current (a constant current of $0.2 \mu \mathrm{mAmp}$ of $6 \mathrm{~V}$ was used for $1 \mathrm{~min}$ bilaterally; Acosta-Galvan et al., 2011).

\section{Data Acquisition}

All data were previously collected and published (AngelesCastellanos et al., 2010; Acosta-Galvan et al., 2011). Locomotion activity data were collected using motion sensors located at the bottom of cages that were designed to continuously monitor the animal motion via the vibration of the cages. Data were collected at 1-min intervals and were re-sampled with the epoch length of 4 min. To minimize transitional artifacts and training effects, data during the first 4 days after the lesion surgery and data during the first 7 days of each food restriction period were excluded from data analysis.

\section{Fractal Analysis}

To assess fractal activity regulation, we examined temporal correlations in activity fluctuations at an array of time scales $\mathrm{n}$ (from 0.1 to $20 \mathrm{~h}$ ). We used the detrended fluctuation analysis (DFA) to quantify the fluctuation amplitude, $F(n)$ at different time scale $\mathrm{n}$ (Peng et al., 1995). A power-law form of $\mathrm{F}(\mathrm{n})$ indicates fractal fluctuations, yielding $\mathrm{F}(\mathrm{n}) \sim \mathrm{n}^{\alpha}$ (a straight line in a log-log plot). The parameter $\alpha$ (the scaling exponent) quantifies the correlation properties in fluctuations: $\alpha=0.5$ indicates no correlation ("white noise"); $\alpha>0.5$ indicates positive correlations. The most interesting, complex behavior is associated with $\alpha \sim 1.0$ which indicates a fine balance between uncorrelated randomness and excessive regularity as observed in healthy physiological outputs (Stanley et al., 1992; Goldberger et al., 2002).

\section{Statistical Analysis}

Results are represented as mean \pm standard error (SE). To assess the effect of time-restricted feeding on fractal activity patterns, ANOVA was performed under each condition (intact, the DHM lesion, and the lesions of both the DMH and the SCN). We compared the results of Group 1 and Group 2 (i.e., between-subjects comparison) to determine the effect of timerestricted feeding in animals with intact DMH and SCN. We compared the results of the same animals in Group 3 under two experimental protocols (i.e., within-subjects comparison) to determine the effect of food restriction in animals after lesioning the DMH or after lesioning the SCN. To study the effect of the lesions, ANOVAs were used to determine the difference among the three conditions for ad libitum and time-restricted feeding, separately. Similar ANOVAs were also performed to assess the effect of food restriction and the lesions on the amplitude of daily activity rhythm.

\section{RESULTS}

\section{Time-Restricted Feeding Induced FAA and Disrupted Fractal Regulation in Intact Animals}

We first examined the influences of time-restricted feeding on intact animals by studying 6 rats with ad libitum food access and 6 rats undergoing a food restriction protocol with a 2-h feeding period at 6-8 h after lights on (Zeitgeber time: ZT 6-8; Figures 1A,B). With ad libitum food access, animals showed a 24-h rhythm in locomotor activity with low levels during the 12$\mathrm{h}$ light phase ( $44 \pm 4 \%$ of daily mean) and high levels during the 12 -h dark phase ( $156 \pm 4 \%$ of daily mean; $p<0.0001$; Figure 1C). Food restriction induced FAA, as characterized by significantly increased activity levels starting $3 \mathrm{~h}$ before the feeding period (at ZT 3-6, food restriction: $141 \pm 4 \%$ of daily mean; ad libitum: $45 \pm$ $6 \%$ of daily mean; $p<0.0001)$ as well as the mean activity level during the entire light phase ( $80 \pm 3 \%$ of daily mean; $p<0.0001$; Figure 1D). Such increased activity during the light phase caused an overall reduced 24 -h rhythm.

In addition to the 24-h rhythm, activity in the intact animals with ad libitum food access exhibited robust fractal fluctuations as characterized by a power-law form of $\mathrm{F}(\mathrm{n}) \sim \mathrm{n}^{\alpha}$ that persisted across a broad range of time scales from minutes up to $12 \mathrm{~h}$ (Figure 2A). Consistent with the previously reported ( $\mathrm{Hu}$ et al., 2007; Hsieh et al., 2014), the scaling exponent ( $\alpha=0.99 \pm$ 0.02 ) was close to 1 , indicating a complex structure with strong temporal correlations in activity fluctuations. Markedly, timerestricted feeding perturbed fractal activity patterns, leading to different correlations in activity fluctuations over two time-scale regions as indicated by the different scaling exponent ( $p=$ $0.0002): \alpha_{1}=1.10 \pm 0.02$ at time scales $<\sim 4 \mathrm{~h}$ (Region I) and $\alpha_{2}=0.85 \pm 0.03$ at time scales $>\sim 4 \mathrm{~h}$ (Region II; Figure 2A). As compared to the results without food restriction, correlations were stronger in Region I (i.e., larger $\alpha_{1} ; p=0.0008$ ) but were much weaker in Region II (i.e., smaller $\alpha_{2}, p=0.013$; Figure 2D). The difference in correlations between two regions remained when excluding the data before and throughout the feeding period (ZT 3-8 h; $p=0.0085$ ).

\section{DMH Lesion Reduced FAA, Disrupted Fractal Regulation, and Reversed the Effect of Food Restriction on Fractal Regulation}

To test whether the $\mathrm{DMH}$ is involved in fractal activity regulation and plays any role in the influence of time-restricted feeding on multiscale activity patterns, we then studied locomotor activity recordings of 6 rats after the lesion of the DMH with ad libitum food (Figure 1A). As compared with intact rats, the DMHlesioned (DMHx) animals maintained the 24-h activity rhythm, showing no significant differences in activity level during the light phase (ZT0-12 h: DMHx: $44 \pm 4 \%$ of daily mean; intact: $44 \pm 4 \%$ of daily mean; $p>0.9$; Figure $1 C$ ). As occurred in the intact animals, food restriction also increased activity levels during the light phase ( $56 \pm 4 \%$ of daily mean; $p=0.0068$ ) and led to an overall reduced circadian rhythmicity in $\mathrm{DMHx}$ 
A

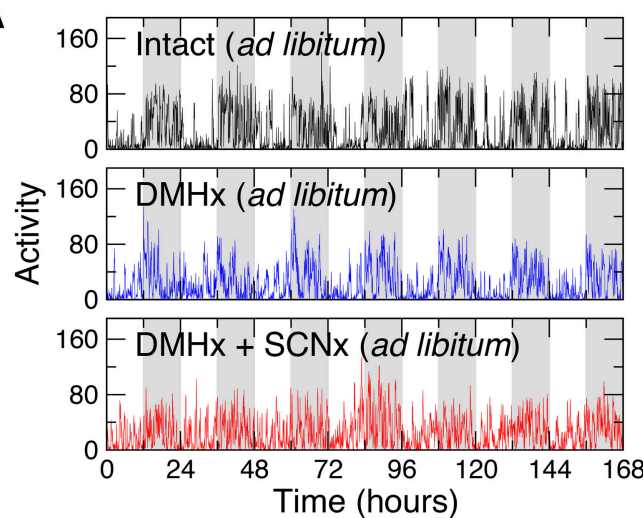

B

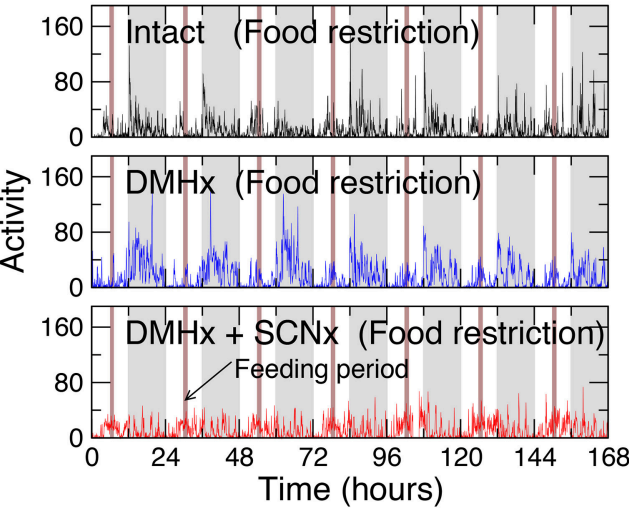

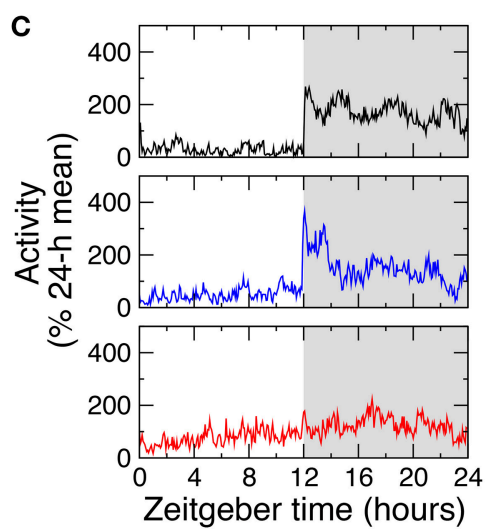

D

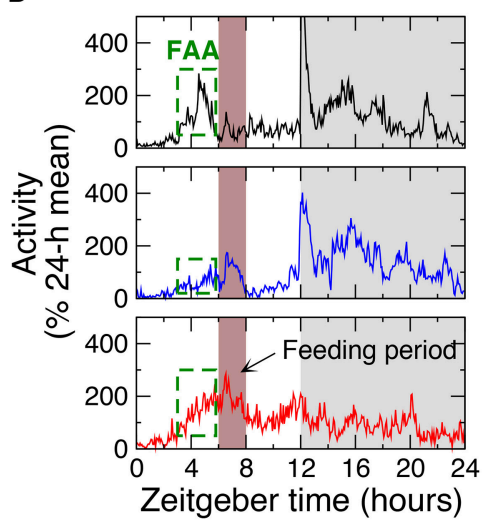

FIGURE 1 | Representative locomotor activity recordings and their averaged daily profiles of rats under $12 \mathrm{~h}: 12 \mathrm{~h}$ light-dark cycles. (A) Recordings of an intact rat, a rat with the lesion of DMH (DMHx rat), and the same DMH-lesion rat after an additional lesion of the SCN with ad libitum food access. Gray (white) bars indicate the dark (light) periods of the light-dark cycle. (B) Recordings of an intact rat and a DMHx rat (the same as in A) before and after the SCN lesion with restricted food availability. Food was available daily for $2 \mathrm{~h}$ at $6-7 \mathrm{~h}$ after light on in each cycle (brown bars). (C) The averaged $24-\mathrm{h}$ activity waveforms of the recordings in (A). (D) The averaged 24-h waveforms of the recordings in (B). To obtain the 24-h activity patterns, data were first normalized by daily means and then averaged across different days. The increased activity levels $3 \mathrm{~h}$ before the feeding period (green dashed boxes) indicate food-anticipatory activity (FAA).

animals (Figure 2D). However, the responses to food restriction in these DMHx animals were much weaker than those in the intact animals, as indicated by the lower activity level during the light phase $(p=0.0009)$. In addition, the increase in the mean activity level at ZT 3-6 (3 h before the feeding time) did not reach a significant level in these DMHx animals (ad libitum: $35 \pm 2 \%$ of daily mean; food restriction: $45 \pm 4 \%$ of daily mean; $p=0.11$ ). Together these results confirmed a reduced FAA in these DMHx animals as previously reported (Gooley et al., 2006; Acosta-Galvan et al., 2011).

Despite the persistence of 24-h activity rhythmicity, lesioning the DMH significantly disrupted fractal activity patterns in a similar manner as the food restriction did to intact animals (Figures 2A,B), i.e., increasing the correlations at time scales $<\sim 4$ h (Region I: $\alpha_{1}=1.06 \pm 0.02 ; p=0.0093$ ) while reducing the correlations at time scales $>\sim 4$ h (Region II $>$ : $\left.\alpha_{2}=0.79 \pm 0.04 ; p=0.0041\right)$. Surprisingly, food restriction had different effects on activity correlations in these DMHx animals as compared to intact animals, i.e., instead of disrupting fractal patterns, food restriction reduced the difference in the correlations between Region I and Region II such that the scaling exponents in the two regions became not significantly different in the DMHx animals (Region I: $\alpha_{1}=1.04 \pm 0.03$; Region II; $\alpha_{2}=0.90 \pm 0.04 ; p=0.08$; Figure 2D). Such "rescuing" effect of food restriction was more pronounced after excluding the data before and throughout the feeding period (ZT 3-8), i.e., $\alpha_{1}$ and $\alpha_{2}$ became virtually the same $\left(\alpha_{1}=1.05 \pm 0.03 ; \alpha_{2}=1.05 \pm 0.03\right.$; $p>0.9)$, suggesting fully restored fractal activity patterns.

\section{Additional Lesion of the SCN Improved FAA and Enhanced the Effect of Time-restricted Feeding on Fractal Regulation}

We further explored the role of the SCN in the influence of timerestricted feeding on motor activity regulation by studying the same $6 \mathrm{DMH}$-lesioned animals after the additional lesion of the SCN (Figures 1A,B). As expected, lesioning the SCN significantly diminished the 24 -h activity rhythm as characterized by a lower normalized power density at $24 \mathrm{~h}(0.07 \pm 0.02 ; p=0.0004$; Figure 1C). Note that the remained 24 -h activity rhythm was likely due to the masking effect of the LD cycles. With the additional lesion of the SCN, time-restricted feeding induced 
A

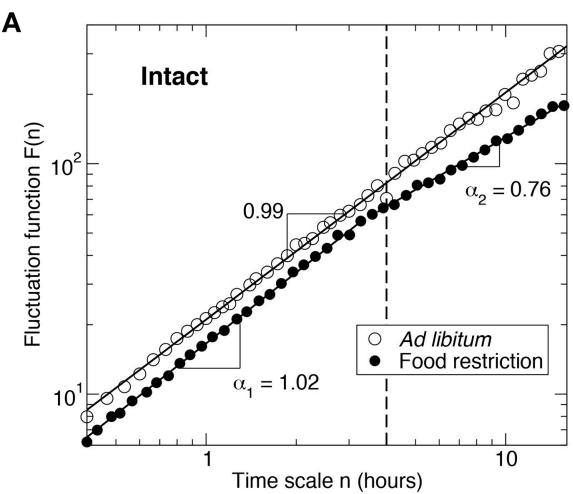

B

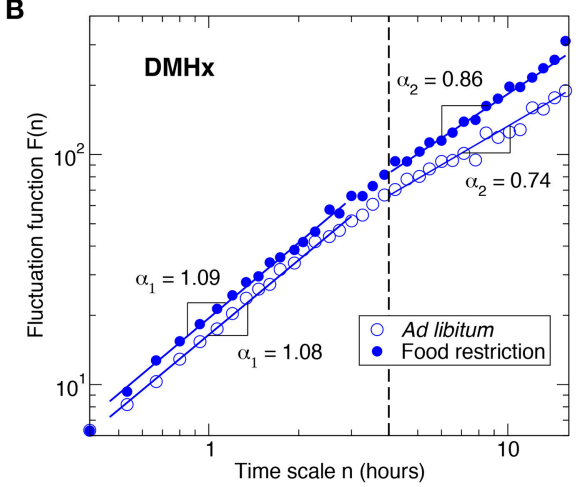

C

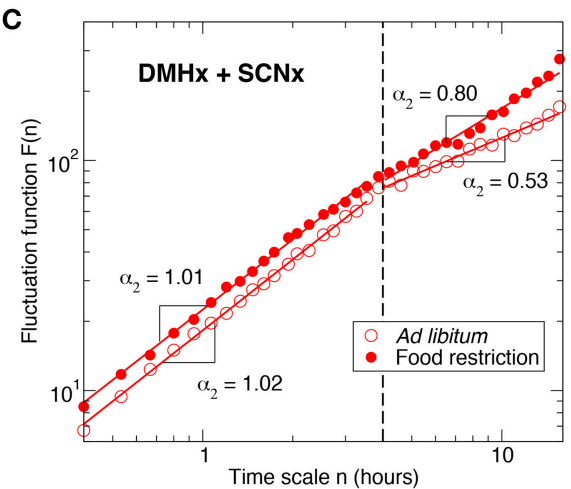

D

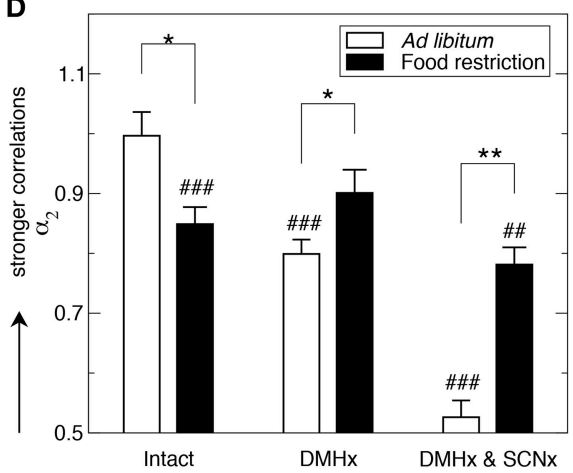

FIGURE 2 | Influence of food restriction on fractal activity patterns in intact animals and in animals after the lesions of the SCN and the DMH. Fluctuation functions of intact animals (A), a rat after the DMH lesion (B), and the same rat (as in B) after the lesions of the DMH and the SCN (C). Data are shown on $\log -\log$ plots. Fluctuation functions are vertically shifted for a better visualization of the influence of food restriction. Results were obtained from the signals shown in Figure 1 using the detrended fluctuation analysis. (D) Scaling exponents at time scales $>4 \mathrm{~h}$. ${ }^{*}$ indicates the effect of food restriction: ${ }^{*} p \leq 0.02$ and ${ }^{* *} p \leq 0.002$. \# indicates the difference between large ( $>4 \mathrm{~h}$ ) and small time scales $(<4 \mathrm{~h}): \#_{p} \leq 0.02, \# \# p \leq 0.002$ and \#\#\# $\leq 0.0002$.

pronounced FAA as indicated by the increased activity level at ZT 3-6 (food restriction: $158 \pm 10 \%$ of daily mean; ad libitum: $85 \pm 7 \%$ of daily mean; $p=0.0012$ ). FAA reversed the 24 -h activeinactive rhythm, leading to higher activity level during the light phase (ZT 0-12: $106 \pm 5 \%$ of daily mean, $p=0.0024$ ) than the level during dark phase (Figure 1D).

Lesioning the SCN further disrupted the fractal activity patterns in these animals (Figure 2C). Specifically, activity fluctuations at time scales $>\sim 4 \mathrm{~h}$ became more random as indicated by a much smaller $\alpha_{2}(0.53 \pm 0.03 ; p<0.0001$; Figure 2D). Consistent with our previous observation in rats with only the lesion of the SCN (Hu et al., 2007), the mean value of $\alpha_{2}$ was not significantly different from 0.5 ( $p>0.3$ ), suggesting the white-noise type of fluctuations without any correlations at $>\sim 4$ h. The correlations in Region I $\left(\alpha_{1}=1.06 \pm 0.02\right)$ remained the same as those before the SCN lesion $(p>0.4)$.

Similar to that observed in these animals before the SCN lesion, time-restricted feeding reduced the difference in correlations between Region I and Region II in the same animals after the SCN lesion (Figures 2C). The main effect was on the correlations in Region II $\left(\alpha_{2}=0.78 \pm 0.04 ; p=0.0015\right.$ for the comparison with ad libitum food). The food-induced increase in $\alpha_{2}$ was greater than the change for these animals with only the lesion of DMH (after the SCNx: $\Delta \alpha_{2}=0.25$; before the SCNx: $\Delta \alpha_{2}=0.11$ ). Despite the "rescuing" effect of time-restricted feeding, $\alpha_{2}$ was still significantly smaller than $\alpha_{1}(1.03 \pm 0.01$; $p=0.0006$; Figure 2). The correlations in Region I were not significantly affected by food restriction $(p>0.3)$.

\section{DISCUSSION}

\section{Food Restriction Impacts Multiscale Activity Control}

Recurring feeding in a fixed, short period during the light phase is widely used to study the anticipatory behavior in animals. However, previous studies have exclusively focused on the change of mean activity levels $1-3 \mathrm{~h}$ before the feeding period in each 24 $\mathrm{h}$ activity cycle. By examining the temporal structure in activity fluctuations, we showed in this study that food restriction with $2 \mathrm{~h}$ of food feeding each day not only induced food anticipatory activity but also disrupted dynamics of motor activity across the entire 24-h cycle and over a wide range of time scales from minutes up to $12 \mathrm{~h}$. The disruption is characterized by the breakdown of fractal patterns in activity fluctuations that were robust in intact animals with ad libitum food access. Importantly, the disruption resembles those observed in animals after the 
lesion of the SCN (Hu et al., 2007) and in humans with dementia and/or Alzheimer's disease ( $\mathrm{Hu}$ et al., 2009, 2013), providing strong evidence that time-restricted feeding during the inactive phase has an adverse impact on motor activity control in intact animals at multiple time scales.

Fractal patterns have been demonstrated in many neurophysiological signals such as heartbeat, gait, respiration, motor activity, and brain activity (Peng et al., 1995, 2002; Hausdorff et al., 1996; Ivanov et al., 2007; Hu et al., 2008a,b; Anteneodo and Chialvo, 2009; He et al., 2010; Fraiman and Chialvo, 2012). One influential interpretation of these fractal physiological fluctuations is the existence of a "long-term memory" in the control system (Stanley et al., 1992; Goldberger et al., 2002; West, 2010a,b). Intuitively, the circadian timing system should participate in memory-keeping because it is responsible for coordinating circadian rhythms in many physiological functions and processes in synchrony with the light-dark cycles (Sakamoto et al., 1998; Weaver, 1998; Yamazaki et al., 2000). Disrupting these circadian oscillations/rhythms and their synchronization, as occurs with aging and in shift work, can lead to deleterious physiological consequences, such as sleep disorders and increased risk for diabetes and cardiovascular diseases (Knutsson et al., 1986; Kawachi et al., 1995; Penev et al., 1998; Schwartz and Roth, 2006; Kroenke et al., 2007; Martino et al., 2007). Thus, the observed disruption in fractal activity patterns under time-restricted feeding may reflect disturbed circadian timing regulation due to two conflicting inputs for circadian timing: one from the light-dark cycle and the other one from the cycle of food-related stimulus (Figure 3). This hypothesis is also consistent with our recent finding that forced activity during the habitual resting phase (light phase: ZT 2-10) could also disrupt fractal activity regulation (Hsieh et al., 2014). Further studies are warranted to test/clarify this theory of memory keeping and circadian timing.

\section{Fractal Regulation Involves both the SCN and the DMH}

It is worth noting that fractal patterns and circadian rhythms represent different properties of the motor control system. Fractal regulation requires an integrated network of multiple control nodes with feedback interactions (Chialvo, 2010). Though the $\mathrm{SCN}$ is a major node in the network for fractal activity control, our previous study showed that the neural network within the SCN cannot generate fractal patterns since the neural activity fluctuations of the SCN in vitro display no fractal patterns $(\mathrm{Hu}$ et al., 2012). In this study we confirm the role of the SCN in fractal activity regulation. We also show that fractal activity patterns can be significantly disrupted despite the persistence of the 24 -h/circadian rhythms as occurred in the animals after the DMH lesion. These results showed that lesions of the $\mathrm{DMH}$ had similar disruptive effects on fractal activity patterns as compared to lesioning only the $\mathrm{SCN}$, leading to more random fluctuations at time scales $>\sim 4 \mathrm{~h}$ and more regular fluctuations at smaller time scales. These results provide direct evidence that the $\mathrm{DMH}$ is also important for the maintenance of fractal activity control (Figure 3A). We note that activity
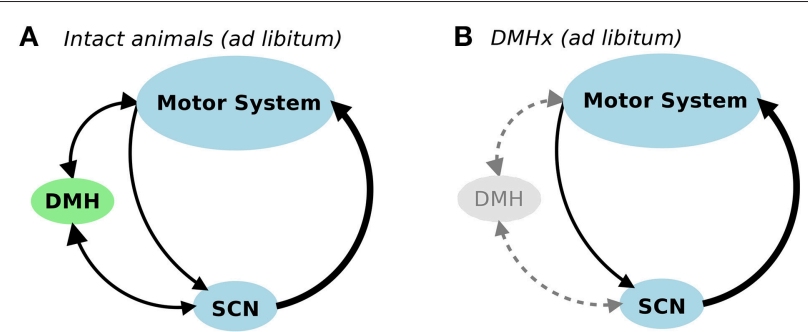

24-h rhythms

Stronge correlations at $>\sim 4 \mathrm{~h}$

24-h rhythms

Reduced correlations at $>\sim 4 \mathrm{~h}$

C Intact animals (food restriction)

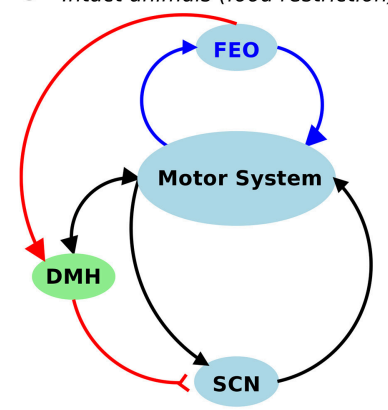

FAA and perturbed 24-h rhythm Reduced correlations at $>\sim 4 \mathrm{~h}$

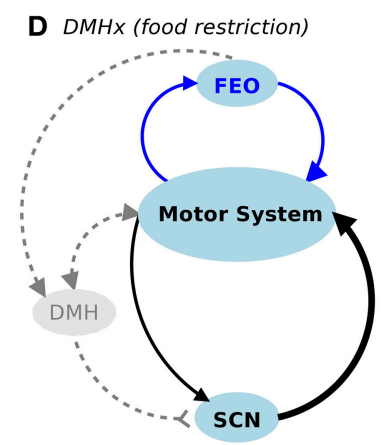

FAA and perturbed 24-h rhythm Restored correlations at $>\sim 4 \mathrm{~h}$

FIGURE 3 | Schematic diagram of the control network for fractal activity regulation. $(\mathbf{A}, \mathbf{B})$ In addition to the $\mathrm{SCN}$, the $\mathrm{DMH}$ is required for fractal activity regulation in intact rats with free access to food. Lesioninng the $\mathrm{DMH}$ led to disrupted fractal regulation as characterized by significantly reduced correlations at $>4 \mathrm{~h}$ (B). (C,D) Restricted food access during the normal inactive periods (light phase of the light-dark cycles) had opposite impacts on fractal activity regulation in intact animals (i.e., causing a reduction in correlations at $>4 \mathrm{~h}$ ) and in $\mathrm{DMH}$-lesioned animals (i.e., causing an increase in correlations at $>4 \mathrm{~h}$ ), despite the similar influences on 24-h activity rhythms (i.e., food-anticipatory activity and reduced amplitude of 24-h rhythm).

fluctuations in the DMH-lesioned animals still possess certain correlations at time scales $>\sim 4 \mathrm{~h}$ (indicated by $\alpha_{2}>0.5$ ), which were completely abolished after the additional lesion of the SCN. These observations suggest that the SCN imparts fractal activity control also through neural nodes other than the $\mathrm{DMH}$ (Figure 3B). This hypothesis is supported by the fact that circadian rhythms in physiological variables, including motor activity and temperature, persist after the DMH lesion.

\section{Effect of Food Restriction on Fractal Regulation}

The most intriguing observations in this study are that food restriction partially rescued the disrupted fractal activity patterns in animals after the lesions of DMH and SCN, and almost fully restored fractal activity in the DMH-lesioned animals (Figures 2A,B). These results have two implications with regard to FAA and fractal activity control.

First, these results suggest that, in addition to SCN-related interactions, there may be food-triggered redundant feedback interactions for the maintenance of fractal activity regulation. Hypothetically these interactions are relevant to the neural circuitry of FAA. It is established that FAA rhythms are only 
present in feeding schedules with periodicity in the circadian range (Stephan, 1981). Since the SCN is not required for FAA, a separate food-entrained oscillator (FEO) is believed to exist within the circadian system. One previous hypothesis was that the FEO is located within the DMH (Chou et al., 2003; Gooley et al., 2006; Mieda et al., 2006) but this hypothesis has been refuted by many other studies, showing persisting FAA after lesioning the DMH (Landry et al., 2006; Mistlberger et al., 2009; Moriya et al., 2009). Despite the current limited understanding of FAA, our results provide evidence that the neural circuitry of FAA may be a key component in the control network for fractal activity regulation at multiple time scales (Figures 3C,D).

Moreover, these results seem to contradict the results from the intact animals (i.e., food restriction disrupted fractal patterns). One possible explanation for this seemingly paradox is that the DMH serves as a "switch," controlling how the information inputs from food restriction and from the light-dark cycles are integrated for fractal activity control. This reminds the role of the DMH in FAA. As we reported in a previous study (AcostaGalvan et al., 2011) and also showed in this study, lesioning the $\mathrm{DMH}$ led to a suppression of FAA while subsequent lesioning the SCN diminished or abolished the suppression. Taken together, the DMH may serve as a double switch for the effects of food restriction on FAA and on fractal activity control: (1) the switch was normally on in intact animals to allow better food anticipation (when food is only available during the inactive phase) while sacrificing other circadian-related processes that are related to fractal activity control (Figure 3C); (2) lesioning the DMH turned off the switch, reducing FAA but allowing the circadian network to integrate the information inputs of food stimulus and of light-dark cycles for better fractal activity control (Figure 3D); and (3) when the SCN was also ablated, the timing of the circadian oscillation was lost such that time-restricted feeding induced more pronounced FAA and improved fractal activity control due to the lack of conflicting or competing time inputs from the SCN. Note that the "conflicting time inputs" could not explain the rescuing effect of time-restricted feeding in the animals after the DMH lesion (but with intact SCN) because daily/circadian rhythms persisted after the DMH lesion. Clearly future studies are required to determine the pathway through which the DMH controls the effect of time-restricted feeding on fractal activity regulation.

\section{LIMITATIONS}

This is the first study demonstrating how time-restricted feeding affects fractal activity regulation. There are a number of notable limitations in this pilot study. First, during the protocol with time-restricted feeding, food was provided at 6-8 h after lights on during the light-dark cycles (i.e., ZT 6-8 h) when animals were normally inactive. Whether restricting feeding at different times can have different impacts on temporal activity correlations is yet to be determined. Specifically, if restricted food is available during the active period or dark phase, there should be no conflicting time cues for activity such that time-restricted feeding may enhance the endogenous circadian activity rhythm, thus not disrupting fractal activity control in control animals while better improving the multiscale control in DMH or SCN-lesioned animals. Second, we focused on fractal activity patterns and did not examine the rhythms at specific time scales except for 24-h rhythm. It is established that rodents exhibit ultradian feeding rhythms of 2-3 h (Gerkema et al., 1993; Bloch et al., 2013). Since the effect of the time-restricted feeding on temporal activity correlations was only pronounced at time scales $>\sim 4$ $\mathrm{h}$, the altered fractal patterns should be not related to potential changes in ultradian feeding rhythms. Third, to examine fractal regulation, we examined only temporal correlations. There are many different statistical properties such as nonlinearity and multifractality that may reveal different aspects of multiscale activity control (Ivanov et al., 1999; Ashkenazy et al., 2001). Finally, many different methods can assess temporal correlations and their performances may be comparable or even better than that of the detrended fluctuation analysis ( $\mathrm{Xu}$ et al., 2005; Makarava et al., 2011). Thus, follow-up studies using different analytical tools are necessary to confirm and explain our observations.

Despite the limitations, this study revealed a previously un-recognized influence of time-restricted food availability on multiscale activity control. Our results provide solid evidence that inducing FAA does not simply impose an event (i.e., feeding period) at a specific circadian time but requires an adjustment in dynamic control of motor activity across the whole circadian cycle and at different time scales. The influence is likely via the impact of food stimulus as an additional time cue (in addition to light inputs) on the circadian network. The different effect of food restriction in the intact animals and in the DHM-lesioned animals indicates that the $\mathrm{DMH}$ plays a crucial role in integrating the timing information from both the SCN and food stimulus. Further studies are warranted to elucidate the interactions among the food-entrained oscillator, the $\mathrm{DMH}$, and the $\mathrm{SCN}$, as well as their mechanistic links to fractal activity regulation.

\section{AUTHOR CONTRIBUTIONS}

$\mathrm{ML}, \mathrm{CE}, \mathrm{RB}$, and $\mathrm{KH}$ conceived and designed the experiments. $\mathrm{RB}$ and $\mathrm{CE}$ performed the experiments and collected data. $\mathrm{ML}, \mathrm{WC}, \mathrm{WH}$, and $\mathrm{KH}$ performed the data analyses. ML and $\mathrm{KH}$ provided analysis tools. $\mathrm{ML}, \mathrm{CE}, \mathrm{RB}$, and $\mathrm{KH}$ wrote the manuscript.

\section{ACKNOWLEDGMENTS}

This research was supported by the National Institutes of Health grants R00-HL102241 and 2P01AG009975 16A1, R01AG04810801A1 (to KH), grants from Ministry of Science and Technology (MOST) in Taiwan MOST 104-3115-E-008-001, MOST 1032321-B-008-003, MOST 103-2221-E-008-006-MY3 (to ML), the grant DGPAPA-UNAM IG 200314 (to RB and CE), and the Consejo Nacional de Ciencia y Tecnología grant, CONACyT 239403 (to CE). 


\section{REFERENCES}

Acosta-Galvan, G., Yi, C.-X., van der Vliet, J., Jhamandas, J. H., Panula, P., Angeles-Castellanos, M., et al. (2011). Interaction between hypothalamic dorsomedial nucleus and the suprachiasmatic nucleus determines intensity of food anticipatory behavior. Proc. Natl. Acad. Sci. U.S.A. 108, 5813-5818. doi: 10.1073/pnas.1015551108

Angeles-Castellanos, M., Salgado-Delgado, R., Rodriguez, K., Buijs, R. M., and Escobar, C. (2010). The suprachiasmatic nucleus participates in food entrainment: a lesion study. Neuroscience 165, 1115-1126. doi: 10.1016/j.neuroscience.2009.11.061

Anteneodo, C., and Chialvo, D. R. (2009). Unraveling the fluctuations of animal motor activity. Chaos 19:033123. doi: 10.1063/1.3211189

Ashkenazy, Y., Ivanov, P. C., Havlin, S., Peng, C. K., Goldberger, A. L., and Stanley, H. E. (2001). Magnitude and sign correlations in heartbeat fluctuations. Phys. Rev. Lett. 86, 1900-1903. doi: 10.1103/PhysRevLett.86.1900

Bloch, G., Barnes, B. M., Gerkema, M. P., and Helm, B. (2013). Animal activity around the clock with no overt circadian rhythms: patterns, mechanisms and adaptive value. Proc. Biol. Sci. 280, 20130019. doi: 10.1098/rspb.2013.0019

Boulos, Z., Rosenwasser, A. M., and Terman, M. (1980). Feeding schedules and the circadian organization of behavior in the rat. Behav. Brain Res. 1, 39-65. doi: 10.1016/0166-4328(80)90045-5

Chialvo, D. R. (2010). Emergent complex neural dynamics. Nat. Phys. 6, 744-750. doi: $10.1038 /$ nphys 1803

Chou, T. C., Scammell, T. E., Gooley, J. J., Gaus, S. E., Saper, C. B., and Lu, J. (2003). Critical role of dorsomedial hypothalamic nucleus in a wide range of behavioral circadian rhythms. J. Neurosci. 23, 10691-10702. Available online at: http://www.jneurosci.org/content/23/33/10691

Escobar, C., Cailotto, C., Angeles-Castellanos, M., Delgado, R. S., and Buijs, R. M. (2009). Peripheral oscillators: the driving force for food-anticipatory activity. Eur. J. Neurosci. 30, 1665-1675. doi: 10.1111/j.1460-9568.2009.06972.x

Fraiman, D., and Chialvo, D. R. (2012). What kind of noise is brain noise: anomalous scaling behavior of the resting brain activity fluctuations. Front. Physiol. 3:307. doi: 10.3389/fphys.2012.00307

Gerkema, M. P., Daan, S., Wilbrink, M., Hop, M. W., and van der Leest, F. (1993). Phase control of ultradian feeding rhythms in the common vole (Microtus arvalis): the roles of light and the circadian system. J. Biol. Rhythms 8, 151-171. doi: 10.1177/074873049300800205

Goldberger, A. L., Amaral, L. A. N., Hausdorff, J. M., Ivanov, P. C., Peng, C.K., and Stanley, H. E. (2002). Fractal dynamics in physiology: alterations with disease and aging. Proc. Natl. Acad. Sci. U.S.A. 99(Suppl. 1), 2466-2472. doi: 10.1073/pnas.012579499

Gooley, J. J., Schomer, A., and Saper, C. B. (2006). The dorsomedial hypothalamic nucleus is critical for the expression of food-entrainable circadian rhythms. Nat. Neurosci. 9, 398-407. doi: 10.1038/nn1651

Hausdorff, J. M., Purdon, P. L., Peng, C. K., Ladin, Z., Wei, J. Y., and Goldberger, A. L. (1996). Fractal dynamics of human gait: stability of long-range correlations in stride interval fluctuations. J. Appl. Physiol. (1985). 80, 1448-1457.

He, B. J., Zempel, J. M., Snyder, A. Z., and Raichle, M. E. (2010). The temporal structures and functional significance of scale-free brain activity. Neuron 66, 353-369. doi: 10.1016/j.neuron.2010.04.020

Hsieh, W.-H., Escobar, C., Yugay, T., Lo, M.-T., Pittman-Polletta, B., SalgadoDelgado, R., et al. (2014). Simulated shift work in rats perturbs multiscale regulation of locomotor activity. J. R. Soc. Interface 11:20140318. doi: 10.1098/rsif.2014.0318

Hu, K., Harper, D. G., Shea, S. A., Stopa, E. G., and Scheer, F. A. J. L. (2013). Noninvasive fractal biomarker of clock neurotransmitter disturbance in humans with dementia. Sci. Rep. 3:2229. doi: 10.1038/srep 02229

Hu, K., Meijer, J. H., Shea, S. A., vanderLeest, H. T., Pittman-Polletta, B., Houben, T., et al. (2012). Fractal patterns of neural activity exist within the suprachiasmatic nucleus and require extrinsic network interactions. PLoS ONE 7:e48927. doi: 10.1371/journal.pone.0048927

Hu, K., Scheer, F. A. J. L., Buijs, R. M., and Shea, S. A. (2008a). The circadian pacemaker generates similar circadian rhythms in the fractal structure of heart rate in humans and rats. Cardiovasc. Res. 80, 62-68. doi: 10.1093/cvr/cvn150

Hu, K., Scheer, F. A. J. L., Buijs, R. M., and Shea, S. A. (2008b). The endogenous circadian pacemaker imparts a scale-invariant pattern of heart rate fluctuations across time scales spanning minutes to 24 hours. J. Biol. Rhythms 23, 265-273. doi: 10.1177/0748730408316166

Hu, K., Scheer, F. A. J. L., Ivanov, P. C., Buijs, R. M., and Shea, S. A. (2007). The suprachiasmatic nucleus functions beyond circadian rhythm generation. Neuroscience 149, 508-517. doi: 10.1016/j.neuroscience.2007.03.058

Hu, K., Van Someren, E. J. W., Shea, S. A., and Scheer, F. A. J. L. (2009). Reduction of scale invariance of activity fluctuations with aging and Alzheimer's disease: involvement of the circadian pacemaker. Proc. Natl. Acad. Sci. U.S.A. 106, 2490-2494. doi: 10.1073/pnas.0806087106

Ivanov, P. C., Amaral, L. A., Goldberger, A. L., Havlin, S., Rosenblum, M. G., Struzik, Z. R., et al. (1999). Multifractality in human heartbeat dynamics. Nature 399, 461-465. doi: 10.1038/20924

Ivanov, P. C., Hu, K., Hilton, M. F., Shea, S. A., and Stanley, H. E. (2007) Endogenous circadian rhythm in human motor activity uncoupled from circadian influences on cardiac dynamics. Proc. Natl. Acad. Sci. U.S.A. 104, 20702-20707. doi: 10.1073/pnas.0709957104

Kalsbeek, A., van Heerikhuize, J. J., Wortel, J., and Buijs, R. M. (1998). Restricted daytime feeding modifies suprachiasmatic nucleus vasopressin release in rats. J. Biol. Rhythms 13, 18-29. doi: 10.1177/074873098128999880

Kawachi, I., Colditz, G. A., Stampfer, M. J., Willett, W. C., Manson, J. E., Speizer, F. E., et al. (1995). Prospective study of shift work and risk of coronary heart disease in women. Circulation 92, 3178-3182. doi: 10.1161/01.CIR.92.11.3178

Knutsson, A., Akerstedt, T., Jonsson, B. G., and Orth-Gomer, K. (1986). Increased risk of ischaemic heart disease in shift workers. Lancet Lond. Engl. 2, 89-92. doi: 10.1016/S0140-6736(86)91619-3

Kroenke, C. H., Spiegelman, D., Manson, J., Schernhammer, E. S., Colditz, G. A., and Kawachi, I. (2007). Work characteristics and incidence of type 2 diabetes in women. Am. J. Epidemiol. 165, 175-183. doi: 10.1093/aje/kwj355

Landry, G. J., Simon, M. M., Webb, I. C., and Mistlberger, R. E. (2006). Persistence of a behavioral food-anticipatory circadian rhythm following dorsomedial hypothalamic ablation in rats. Am. J. Physiol. Regul. Integr. Comp. Physiol. 290, R1527-R1534. doi: 10.1152/ajpregu.00874.2005

Makarava, N., Benmehdi, S., and Holschneider, M. (2011). Bayesian estimation of self-similarity exponent. Phys. Rev. E Stat. Nonlin. Soft Matter Phys. 84:021109. doi: 10.1103/PhysRevE.84.021109

Martino, T. A., Tata, N., Belsham, D. D., Chalmers, J., Straume, M., Lee, P., et al. (2007). Disturbed diurnal rhythm alters gene expression and exacerbates cardiovascular disease with rescue by resynchronization. Hypertension 49, 1104-1113. doi: 10.1161/HYPERTENSIONAHA.106.083568

Merkestein, M., van Gestel, M. A., van der Zwaal, E. M., Brans, M. A., Luijendijk, M. C., van Rozen, A. J., et al. (2014). GHS-Rla signaling in the DMH and VMH contributes to food anticipatory activity. Int. J. Obes. 38, 610-618. doi: 10.1038/ijo.2013.131

Mieda, M., Williams, S. C., Richardson, J. A., Tanaka, K., and Yanagisawa, M. (2006). The dorsomedial hypothalamic nucleus as a putative food-entrainable circadian pacemaker. Proc. Natl. Acad. Sci. U.S.A. 103, 12150-12155. doi: 10.1073/pnas.0604189103

Mistlberger, R. E. (1994). Circadian food-anticipatory activity: formal models and physiological mechanisms. Neurosci. Biobehav. Rev. 18, 171-195. doi: 10.1016/0149-7634(94)90023-X

Mistlberger, R. E., Buijs, R. M., Challet, E., Escobar, C., Landry, G. J., Kalsbeek, A., et al. (2009). Standards of evidence in chronobiology: critical review of a report that restoration of Bmall expression in the dorsomedial hypothalamus is sufficient to restore circadian food anticipatory rhythms in Bmal1-/- mice. J. Circadian Rhythms 7:3. doi: 10.1186/1740-3391-7-3

Mistlberger, R. E., Kent, B. A., Chan, S., Patton, D. F., Weinberg, A., and Parfyonov, M. (2012). Circadian clocks for all meal-times: anticipation of 2 daily meals in rats. PLoS ONE 7:e31772. doi: 10.1371/journal.pone.0031772

Mistlberger, R. E., and Marchant, E. G. (1995). Computational and entrainment models of circadian food-anticipatory activity: evidence from non-24hr feeding schedules. Behav. Neurosci. 109, 790-798. doi: 10.1037/07357044.109.4.790

Moriya, T., Aida, R., Kudo, T., Akiyama, M., Doi, M., Hayasaka, N., et al. (2009). The dorsomedial hypothalamic nucleus is not necessary for food-anticipatory circadian rhythms of behavior, temperature or clock gene expression in mice. Eur. J. Neurosci. 29, 1447-1460. doi: 10.1111/j.1460-9568.2009.06697.x

Patton, D. F., Katsuyama, A. M., Pavlovski, I., Michalik, M., Patterson, Z., Parfyonov, M., et al. (2014). Circadian mechanisms of food anticipatory 
rhythms in rats fed once or twice daily: clock gene and endocrine correlates. PLoS ONE 9:e112451. doi: 10.1371/journal.pone.0112451

Penev, P. D., Kolker, D. E., Zee, P. C., and Turek, F. W. (1998). Chronic circadian desynchronization decreases the survival of animals with cardiomyopathic heart disease. Am. J. Physiol. 275, H2334-H2337.

Peng, C. K., Havlin, S., Hausdorff, J. M., Mietus, J. E., Stanley, H. E., and Goldberger, A. L. (1995). Fractal mechanisms and heart rate dynamics. J. Electrocardiol. 28(Suppl.) 59-65. doi: 10.1016/s0022-0736(95) 80017-4

Peng, C. K., Mietus, J. E., Liu, Y., Lee, C., Hausdorff, J. M., Stanley, H. E., et al. (2002). Quantifying fractal dynamics of human respiration: age and gender effects. Ann. Biomed. Eng. 30, 683-692. doi: 10.1114/1.14 81053

Pittman-Polletta, B. R., Scheer, F. A. J. L., Butler, M. P., Shea, S. A., and Hu, K. (2013). The role of the circadian system in fractal neurophysiological control. Biol. Rev. 88, 873-894. doi: 10.1111/brv.12032

Reppert, S. M., and Weaver, D. R. (2002). Coordination of circadian timing in mammals. Nature 418, 935-941. doi: 10.1038/nature00965

Sakamoto, K., Nagase, T., Fukui, H., Horikawa, K., Okada, T., Tanaka, H., et al. (1998). Multitissue circadian expression of rat period homolog (rPer2) mRNA is governed by the mammalian circadian clock, the suprachiasmatic nucleus in the brain. J. Biol. Chem. 273, 27039-27042. doi: 10.1074/jbc.273.42. 27039

Schwartz, J. R. L., and Roth, T. (2006). Shift work sleep disorder: burden of illness and approaches to management. Drugs 66, 2357-2370. doi: 10.2165/00003495200666180-00007

Silver, R., Balsam, P. D., Butler, M. P., and LeSauter, J. (2011). Food anticipation depends on oscillators and memories in both body and brain. Physiol. Behav. 104, 562-571. doi: 10.1016/j.physbeh.2011.05.034

Stanley, H. E., Buldyrev, S. V., Goldberger, A. L., Hausdorff, J. M., Havlin, S., Mietus, J., et al. (1992). Fractal landscapes in biological systems: long-range correlations in DNA and interbeat heart intervals. Phys. A 191, 1-12. doi: 10.1016/0378-4371(92)90497-E
Stephan, F. (1981). Limits of entrainment to periodic feeding in rats with suprachiasmatic lesions. J. Comp. Physiol. 143, 401-410. doi: 10.1007/BF006 09906

Stephan, F. K. (2002). The "other" circadian system: food as a Zeitgeber. J. Biol. Rhythms 17, 284-292. doi: 10.1177/074873002129002591

Takasu, N. N., Kurosawa, G., Tokuda, I. T., Mochizuki, A., Todo, T., and Nakamura, W. (2012). Circadian regulation of food-anticipatory activity in molecular clock-deficient mice. PLoS ONE 7:e48892. doi: 10.1371/journal.pone.0048892

Weaver, D. R. (1998). The suprachiasmatic nucleus: a 25 -year retrospective. J. Biol. Rhythms 13, 100-112. doi: 10.1177/074873098128999952

West, B. J. (2010a). Fractal physiology and the fractional calculus: a perspective. Front. Physiol. 1:12. doi: 10.3389/fphys.2010.00012

West, B. J. (2010b). The wisdom of the body; a contemporary view. Front. Physiol. 1:1. doi: 10.3389/fphys.2010.00001

Xu, L. M., Ivanov, P. C., Hu, K., Chen, Z., Carbone, A., and Stanley, H. E. (2005). Quantifying signals with power-law correlations: a comparative study of detrended fluctuation analysis and detrended moving average techniques. Phys. Rev. E 71:051101. doi: 10.1103/physreve.71.051101

Yamazaki, S., Numano, R., Abe, M., Hida, A., Takahashi, R., Ueda, M., et al. (2000). Resetting central and peripheral circadian oscillators in transgenic rats. Science 288, 682-685. doi: 10.1126/science.288.5466.682

Conflict of Interest Statement: The authors declare that the research was conducted in the absence of any commercial or financial relationships that could be construed as a potential conflict of interest.

Copyright $\odot 2016$ Lo, Chiang, Hsieh, Escobar, Buijs and Hu. This is an open-access article distributed under the terms of the Creative Commons Attribution License (CC $B Y)$. The use, distribution or reproduction in other forums is permitted, provided the original author(s) or licensor are credited and that the original publication in this journal is cited, in accordance with accepted academic practice. No use, distribution or reproduction is permitted which does not comply with these terms. 